\title{
OBSERVATIONS OF THE HIGH-MASS X-RAY BINARY A 0535+26 IN QUIESCENCE
}

\author{
Richard Rothschild $^{1}$, Alex Markowitz $^{1,2,8}$, Paul Hemphill $^{1}$, Isabel Caballero $^{3}$, Katja Pottschmidt $^{4}$, \\ Matthias Kühnel ${ }^{2}$, Jörn Wilms ${ }^{2}$, Felix Fürst ${ }^{5}$, Victor Doroshenko ${ }^{6}$, and Ascension CAMEro-Arranz ${ }^{7}$ \\ ${ }^{1}$ University of California, San Diego, Center for Astrophysics and Space Sciences, 9500 Gilman Dr., La Jolla, CA 92093-0424, USA; rrothschild@ucsd.edu \\ ${ }^{2}$ Dr. Karl-Remeis-Sternwarte and ECAP, Sternwartstr. 7, D-96049 Bamberg, Germany \\ ${ }^{3}$ CEA Saclay, DSM/IRFU/SAp -UMR AIM (7158) CNRS/CEA/Université P. Diderot, Orme des Merisiers, Bat. 709, F-91191 Gif-sur-Yvette, France \\ ${ }^{4}$ CRESST, UMBC, and NASA GSFC, Code 661, Greenbelt, MD 20771, USA \\ ${ }^{5}$ Space Radiation Lab, MC 290-17 Cahill, California Institute of Technology, 1200 E. California Blvd, Pasadena, CA 91125, USA \\ ${ }^{6}$ Institut für Astronomie und Astrophysik, Universität Tübingen, Sand 1, D-72076 Tübingen, Germany \\ ${ }^{7}$ Institut de Ciències de l'Espai, (IEEC-CSIC), Campus UAB, Fac. de Ciències, Torre C5, parell, 2a planta, E-08193 Barcelona, Spain \\ Received 2013 January 10; accepted 2013 April 23; published 2013 May 20
}

\begin{abstract}
We have analyzed three observations of the high-mass X-ray binary A $0535+26$ performed by the Rossi X-Ray Timing Explorer (RXTE) three, five, and six months after the last outburst in 2011 February. We detect pulsations only in the second observation. The 3-20 keV spectra can be fit equally well with either an absorbed power law or absorbed thermal bremsstrahlung model. Reanalysis of two earlier RXTE observations made $4 \mathrm{yr}$ after the 1994 outburst, original BeppoSAX observations 2 yr later, reanalysis of four EXOSAT observations made $2 \mathrm{yr}$ after the last 1984 outburst, and a recent XMM-Newton observation in 2012 reveal a stacked, quiescent flux level decreasing from $\sim 2$ to $<1 \times 10^{-11} \mathrm{erg} \mathrm{cm}^{-2} \mathrm{~s}^{-1}$ over $6.5 \mathrm{yr}$ after outburst. The detection of pulsations during half of the quiescent observations would imply that accretion onto the magnetic poles of the neutron star continues despite the fact that the circumstellar disk may no longer be present. The accretion could come from material built up at the corotation radius or from an isotropic stellar wind.
\end{abstract}

Key words: pulsars: general - stars: individual (A 0535+26) - stars: neutron - X-rays: stars

Online-only material: color figure

\section{INTRODUCTION}

A $0535+26$ is a Be/X-ray binary system, discovered by Ariel $V$ during a giant outburst in 1975 (Rosenberg et al. 1975). The binary system consists of the neutron star pulsar A0535+26 and O9.7-B0 IIIe optical companion HDE 245770 (Steele et al. 1998). The neutron star moves in an eccentric orbit with $e=$ 0.47 , an orbital period $P_{\text {orb }}=111.07 \pm 0.07$ days, and exhibiting a pulse period of $\sim 103.25 \mathrm{~s}$ (Camero-Arranz et al. 2012 and references therein). Its estimated distance to the system is $d=$ $2 \mathrm{kpc}$ (Steele et al. 1998). Extensive reviews of the system are given in Giovannelli \& Graziati (1992) and Caballero (2009). A $0535+26$ belongs to a class of high-mass systems, called $\mathrm{Be} / \mathrm{X}$-ray binaries, known for having outbursts where matter accretes onto the magnetic poles via an accretion disk that is filled at periastron passage from a circumstellar decretion disk of the primary. When this stellar disk retreats, the accretion disk shrinks and the source enters quiescence. This allows the observer to study other source(s) of accretion that may be present. Campana et al. (2002) studied high-mass systems in quiescence where the neutron star spin period is relatively short $\left(P_{\text {spin }}<5 \mathrm{~s}\right)$, and they conclude that X-ray emission is due to material falling onto the magnetospheric boundary or burning on the neutron star surface. Longer period high-mass systems are less well studied.

The X-ray intensity of A $0535+26$ varies by almost three orders of magnitude with three basic intensity states: (1) quiescence, (2) normal, or type I, outbursts, generally associated with periastron passages, and (3) giant, or type II, outbursts that may occur at any orbital phase. The companion, HDE 245770, has an equatorial circumstellar disk whose size has varied over time.

\footnotetext{
8 Alexander von Humboldt Fellow.
}

It is this material that drives the normal outbursts at periastron. The giant outbursts may arise from large asynchronous mass outflows from the companion. An indicator of the disk size is the $\mathrm{H} \alpha$ equivalent width, as well as that of $\mathrm{He}$ I and the overall visual magnitude (Grundstrom et al. 2007). The results of monitoring of these quantities are given in Figure 1 of CameroArranz et al. (2012) for the past $37 \mathrm{yr}$. A large reduction in the $\mathrm{H} \alpha$ strength occurred in 1998 (MJD 51000) in conjunction with the ceasing of outbursts for 7 yr. New outbursts began anew with the giant outburst of 2005 (Figure 1, left). Beginning in 2009 , the $\mathrm{H} \alpha$ strength again declined with an apparent levelingoff at the time of the 2011 February outburst. Since then the $\mathrm{H} \alpha$ strength has continued to decline (A. Camero-Arranz 2012, private communication) and no outbursts have been detected.

Since its discovery, nine giant outbursts have been detected: in 1977 December (Giovannelli \& Graziati 1992), 1980 October (Nagase et al. 1982), 1983 June (Sembay et al. 1990), 1989 March/April (Makino et al. 1989), 1994 February (Finger et al. 1994), 2005 May/June (Tueller et al. 2005), 2009 December (Wilson-Hodge et al. 2009), 2010 October (Mihara et al. 2010), and 2011 February (Camero-Arranz et al. 2012). Normal outbursts are seen at periastron passages (see Figure 4 in Motch et al. 1991), but may not occur for years at a time. After the 1998 normal outburst, the source went into the quiescent state until resuming in 2005 (Figure 1, left). The last outburst was the giant one in 2011 February. Since then, A0525+26 has exhibited no outbursts at all (Figure 1, right).

In quiescence, the system is expected to be in the centrifugally inhibited regime (Illarionov \& Sunyaev 1975), preventing the continual accretion of matter onto the neutron star. Nevertheless, all historic observation campaigns during quiescence have found pulsations in at least part of their data, indicating that matter is still being accreted along the magnetic field lines. Observations 

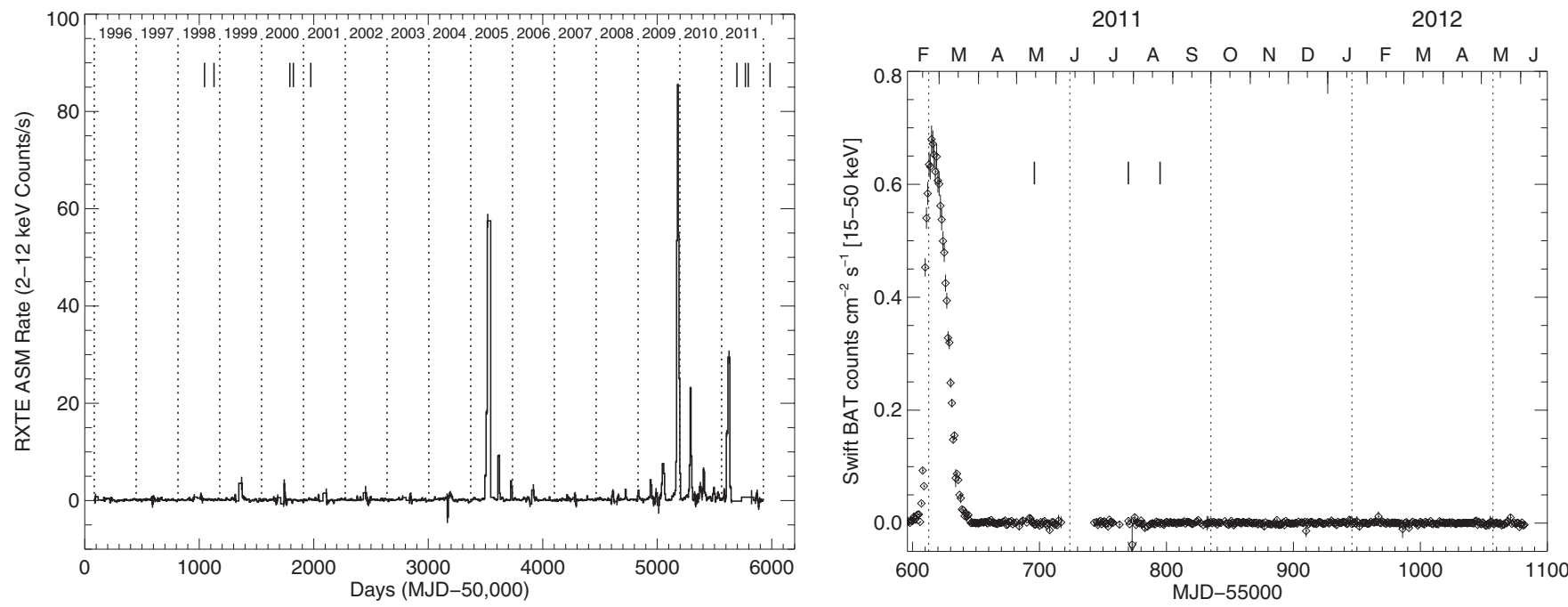

Figure 1. (Left) RXTE/ASM 2-12 keV weekly light curve for the transient A $0535+26$ throughout the RXTE mission and (Right) the Swift/BAT 15-50 keV daily light curve from 2011 to present (bottom). Yearly boundaries are shown by vertical dotted lines in the plot on the left, and periastron passages are marked by vertical dotted lines in the plot on the right. The dates of the RXTE, XMM-Newton, and BeppoSAX observations are noted by short vertical lines in the plot on the left, and the three new RXTE pointed observations are marked on the plot on the right. See Table 2 for dates and observatories. No outbursts have been seen since the 2011 February outburst.

of the quiescent state have been performed by EXOSAT, RXTE, BeppoSAX, and XMM-Newton. The source was observed four times by EXOSAT between 1985 and 1986 and pulsations were detected during two of the observations (Motch et al. 1991). RXTE performed two observations in quiescence between 1998 August and 1998 November (Negueruela et al. 2000), and pulsations were detected during the 1998 November observation, while in August only a weak indication $(<3 \sigma)$ was found for periodicity at the nominal pulse period. BeppoSAX observed the source during quiescence in 2000 September-October and 2001 March (Orlandini et al. 2004). Mukherjee \& Paul (2005) divided the BeppoSAX data into five ever-decreasing count-rate bins, and significantly detected pulsations in all but the faintest.

We report here on three $R X T E$ observations made in 2011 after the giant outburst of 2011 February, and pulsations were only detected in the second of the new observations. Combining these three observations with the previous observations and a new XMM-Newton observation made a year after the last outburst (V. Doroshenko 2012, private communication), we present a detailed study of A0535+26 in quiescence, where accretion onto the magnetic poles continues, possibly from matter accumulated at the magnetospheric radius or a stellar wind. Section 2 describes the data reduction and analysis, while Section 3 discusses the results and presents the conclusions of this investigation.

\section{DATA REDUCTION AND ANALYSIS}

RXTE observed A $0535+26$ in quiescence on three occasions in 2011 in order to study its spectral and temporal behavior (Table 1). The data reduction was carried out using HEASOFT version 6.11 software, and XSPEC version 12.7.0 (Arnaud 1996). The data were extracted observing the standard UCSD/IAAT/ECAP filtering criteria of pointing within $0.01 \mathrm{deg}$ of the target, elevation above the Earth's horizon greater than $10^{\circ}$, time since the center of the last South Atlantic Anomaly passage of 30 minutes, and an electron rate of less than 0.1 .

We have also reanalyzed the $1998 R X T E$ observations of A $0535+26$ reported by Negueruela et al. (2000) and the 1985-1986 EXOSAT observations of Motch et al. (1991)
Table 1

Observations of A $0535+26$ with $R X T E$

\begin{tabular}{lcccc}
\hline \hline Date & ObsID & Rate $^{\mathrm{a}, \mathrm{b}}$ & Lvt $^{\mathrm{c}}$ & Phase $^{\mathrm{d}}$ \\
\hline 2011 May 15 & $96421-01-02-00$ & $3.9 \pm 0.1$ & 2,432 & 0.749 \\
2011 Jul 28 & $96421-01-03-00$ & $2.3 \pm 0.1$ & 2,944 & 0.415 \\
2011 Aug 22 & $96421-01-04-00$ & $1.4 \pm 0.1$ & 2,672 & 0.640
\end{tabular}

Notes.

a PCU2 3-90 keV background subtracted counting rate in counts $\mathrm{s}^{-1}$.

${ }^{\mathrm{b}}$ Uncertainties in rates are $68 \%$ confidence.

c The livetime in seconds.

${ }^{\mathrm{d}}$ Based upon $P_{\text {orb }}=111.1$ days, MJD $($ Periastron $)=53613.0$.

using updated response and background files. ${ }^{9}$ The reanalysis of the EXOSAT spectra for A $0535+26$ was performed by downloading the spectral and response files for the EXOSAT ME detector from the HEASARC for the four observations. The XMM-Newton results will be the subject of a separate publication, and only the $2-10 \mathrm{keV}$ flux is presented here. The BeppoSAX results are from the publications of Orlandini et al. (2004) and Mukherjee \& Paul (2005). In addition, we have used the abundances of Wilms et al. (2000) and cross sections of Verner et al. (1996). Table 2 summarizes the 13 observations made in quiescence since 1984 in order of their time since the last outburst preceding them.

\subsection{Timing}

To perform a period search, Proportional Counter Unit 2 (PCU2; Jahoda et al. 2006) light curves from the observations in 2011 July (ObsID 96421-01-03-00) and 2011 August (ObsID 96421-01-04-00) were extracted in data mode B_250ms_128M and the light curve from the observation in 2011 May (ObsID 96421-01-02-00) in GoodXenon mode. Due to the low luminosity of the source, no significant flux was detected above $\sim 20 \mathrm{keV}$, and therefore only data from pulse height channels 5-49, corresponding to energies between $\sim 3$ and $\sim 20 \mathrm{keV}$, were used in the timing analysis (see Table 1 for

\footnotetext{
9 Our reanalysis appears to show that Motch et al. (1991) have a typographical error in their Table 2-the 1-10 keV flux should be $\times 10^{-11} \mathrm{erg} \mathrm{cm}^{-2} \mathrm{~s}^{-1}$.
} 
Table 2

Quiescent Observations of A $0535+26$

\begin{tabular}{|c|c|c|c|c|c|}
\hline Date & Satellite & MJD & $\Delta \mathrm{T}^{\mathrm{a}}$ & Phase $^{b}$ & Pulses? \\
\hline 2011 May 15 & $R X T E$ & 55696 & 77 & 0.749 & No \\
\hline 2011 Jul 28 & $R X T E$ & 55770 & 155 & 0.415 & Yes \\
\hline 2011 Aug 22 & $R X T E$ & 55795 & 180 & 0.640 & No \\
\hline 2012 Feb 28 & $X M M$ & 55985 & 370 & 0.350 & Yes \\
\hline 1985 Sep 23 & EXOSAT & 46331 & 600 & 0.545 & Yes \\
\hline 1986 Feb 10 & EXOSAT & 46471 & 740 & 0.284 & No \\
\hline 1986 Feb 18 & EXOSAT & 46479 & 748 & 0.212 & Yes \\
\hline 1986 Mar 9 & EXOSAT & 46498 & 767 & 0.041 & No \\
\hline 1998 Aug 21 & $R X T E$ & 51046 & 1455 & 0.105 & Yes \\
\hline 1998 Nov 12 & $R X T E$ & 51129 & 1538 & 0.358 & Yes \\
\hline 2000 Sep 4-5 & ВерроSAX & 51791 & 2200 & 0.400 & No \\
\hline 2000 Oct 5-6 & ВерроSAX & 51822 & 2231 & 0.121 & No \\
\hline 2001 Mar 6-7 & ВерроSAX & 51974 & 2383 & 0.753 & Yes \\
\hline
\end{tabular}

Notes.

a Days since the peak of the previous outburst; 2011 February 23, 1994 August 27, or 1984 February 1.

b Based upon $P_{\text {orb }}=111.1$ days, MJD (Periastron $)=53613.0$.

the comparative counting rates). For each observation the data were collected into consecutive $2 \mathrm{~s}$ time bins. With uniformly sampled and uninterrupted data, the Lomb-Scargle algorithm was applied over the frequency range of $0-0.020 \mathrm{~Hz}$ (periods greater than $50 \mathrm{~s}$ ) in 160 frequency steps (approximately $1 \mathrm{~s}$ steps in period). The resulting Lomb-Scargle significance is plotted versus frequency for the three data sets in Figure 2 (left column). The probability of a frequency component occurring in pure Gaussian noise is $\operatorname{Pr}(\mathrm{occ}) \cong(0.01)^{s / 4.6}$, where $s$ is the significance. Thus a significance of 66.7 , for the middle observation at $0.0097 \mathrm{~Hz}$, implies a very significant detection. The interpretation of the actual significance is limited by systematic effects, such as aliasing and non-Gaussian variations. The peak of the Lomb-Scargle plot for the middle observation was at 102.36 s. Note that the Lomb-Scargle analysis assumes sinusoidal variations and Gaussian noise, while the pulse profile of A $0535+26$ is somewhat sinusoidal (lower right panel of Figure 2) and the statistics are Poisson. Epoch folding for periods near the Lomb-Scargle peak value was used to further define the pulse period. The first and last observations produce very little Lomb-Scargle significance near $0.0097 \mathrm{~Hz}(103 \mathrm{~s})$, and we conclude that pulsing was not detected for those observations. Epoch folding for those data was performed to validate the Lomb-Scargle analysis.

An iterative epoch folding method was utilized whereby pulse-to-pulse changes in luminosity were taken into account by averaging the count rate over the pulse period and subtracting the resulting pulse averaged light curve from the original one. This increases the strength of a signal in the epoch folding $\chi^{2}$-distribution (Larsson 1996). Periodic signals were searched between 94 and $112 \mathrm{~s}$, with 200 test periods in between and 8 phase bins. The resulting $\chi^{2}$ distribution for the 2011 July observation is shown in Figure 2 (top right panel), and the corresponding 16 bin folded light curve for a peak period of $103.48(28) \mathrm{s}$ is given on the middle right panel. If one compares the second observation profile to those seen in outburst (e.g., Caballero et al. 2007), this 3-20 keV profile resembles the higher energy ( $>15 \mathrm{keV}$ ) ones in outburst with the broader minimum, as compared to lower energy ones with a narrow feature defining flux minimum. It also resembles many of the previous quiescent $2-10 \mathrm{keV}$ profiles. The uncertainty in the epoch fold period for the 2011 July data is at the $1 \sigma$-confidence limit and was determined by Monte Carlo simulations. The 2011 July pulse period is consistent with the detected pulse period of $\sim 103.25 \mathrm{~s}$ (Camero-Arranz et al. 2012) during the last outburst in February 2011, and considering that the pulse period changes relatively little during non-outburst times. It is surprising that the pulsations were only detected in the 2011 July observation, which has an intermediate flux of the three observations. Even if the lack of a detection of pulsations in the last of the three observations could be attributed to insufficient statistics, the non-detection in the brightest observation remains puzzling. With exposure roughly equivalent to the 2011 July observation and higher counting rate, one would expect it to have been more sensitive to pulsations. The energy spectra of all three observations are quite similar (Figure 3), so the presence or lack of pulsations does not appear attributable to some kind of state change.

\subsection{Spectra}

The spectra for each $R X T E$ observation were generated from the PCU2 Standard2 Data files, which represent the full (2-90 keV) energy range in 129 variable width spectral bins. These histograms are independent of the individualized data modes for a given observation. The PCU2 Faint background estimate, provided by the Proportional Counter Array (PCA) team and based upon detector rates acquired while observing blank fields during the mission, was used in fitting of background subtracted data. The normalization of the background histogram depends upon the dead time estimate, which is also based upon average detector counting rates. The XSPEC recorn function was initially used to correct for the small difference between the estimated and actual PCU2 background normalization, which cannot be measured directly due to the PCA detectors being non-imaging. Consequently, the background must be modeled. Comparison of results with and without the recorn function in the model showed that these data were insensitive to this background optimization, and thus results are given for fits without the recorn function included in the modeling. The two spectral models fitted to the three observations in the 3-20 keV range were an absorbed power law (CFLUX *(TBABS*PEGPWRLW)) and absorbed thermal bremsstrahlung (CFLUX*(TBABS*BREMSS)), both modified by a maximum in Galactic line of sight absorption (fixed at $4.5 \times 10^{21} \mathrm{~cm}^{-2}$, Kalberla et al. 2005). CFLUX was the basis for the measurement of $2-10 \mathrm{keV}$ flux plus $90 \%$ errors. The simple power law was used since the statistics did not warrant the use of a more complex model with a high-energy cutoff, as utilized for fitting outburst spectra. Both models gave acceptable and similar fits (Figure 3, Tables 3 and 4). The errors reported in the two tables are $90 \%$ uncertainties. The powerlaw and thermal bremsstrahlung best-fit values for absorption at the source differ by about a factor of two, with the powerlaw one averaging about $9 \times 10^{22} \mathrm{~cm}^{-2}$ and bremsstrahlung one averaging a bit less than $4 \times 10^{22} \mathrm{~cm}^{-2}$. This is due to the curvature of the bremsstrahlung spectrum with respect to that of a power law at low energies. Above $\sim 10 \mathrm{keV}$, the bremsstrahlung spectrum rolls over with respect to the power law, but poor statistics at these energies preclude the determination of which model provides the better representation of the incident spectrum.

The 1998 RXTE spectral data for A $0535+26$ were extracted with the same acceptance criteria as the 2011 RXTE data, and reanalyzed with updated response and background files. For the present RXTE reanalysis, only data from PCU2 were used for 

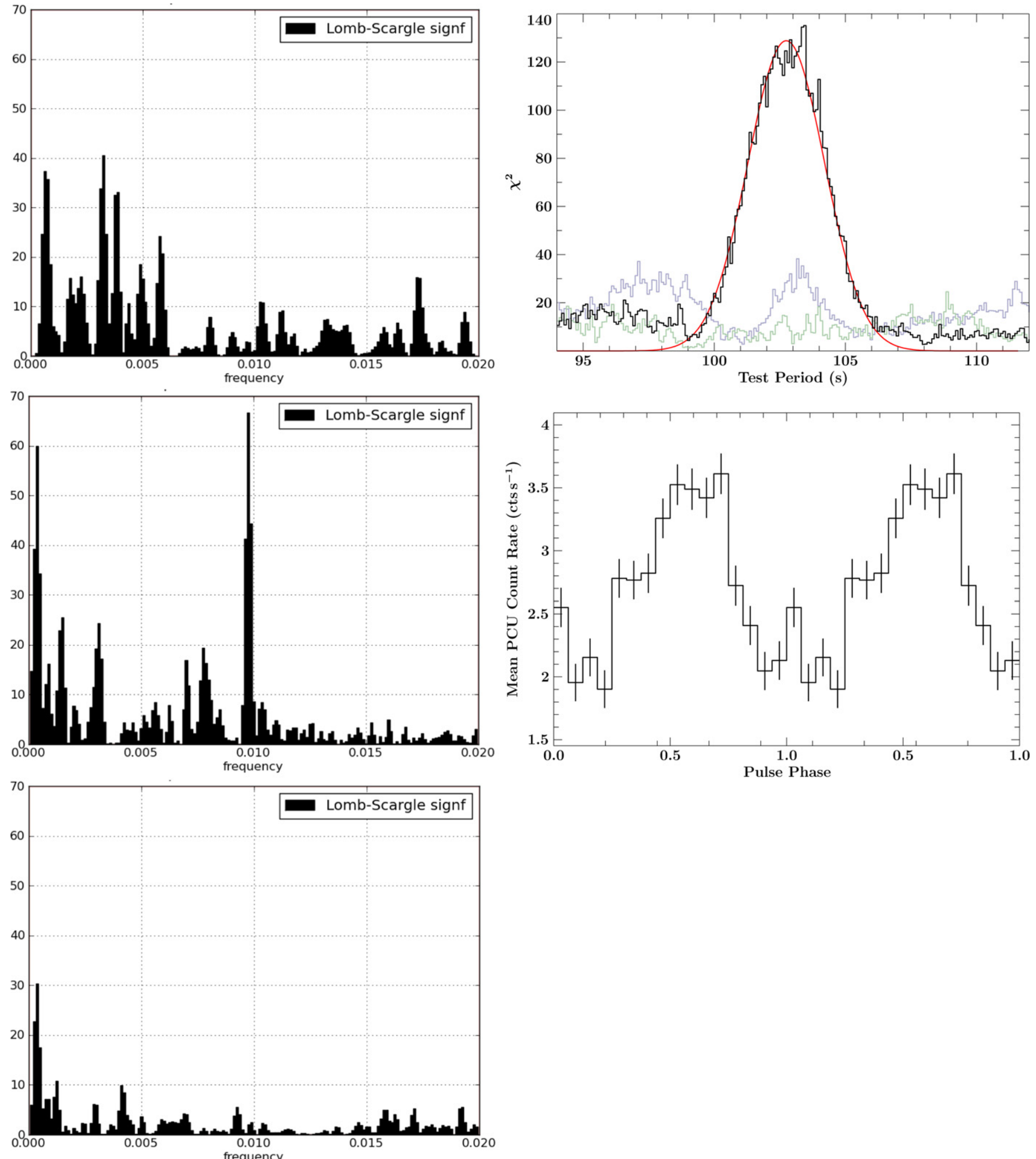

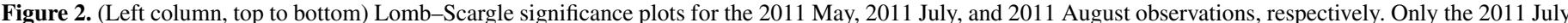

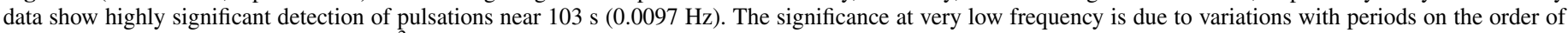

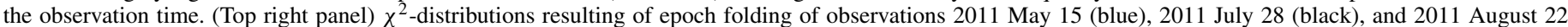

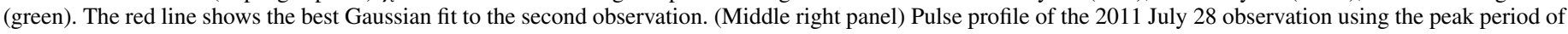
103.41(28) s. The profile is repeated once to enhance visibility.

(A color version of this figure is available in the online journal.)

consistency with the later RXTE results. Only $2 \sigma$ upper limits to the column density were found, and the best-fit values of the power-law indices were less than, but consistent with, those found by Negueruela et al. (2000). When applying the bremsstrahlung model, the column density upper limits were again lower than those for the power-law fits, and the electron temperatures were somewhat higher, but again consistent with the values found by Negueruela et al. (2000). Tables 3 and 4 

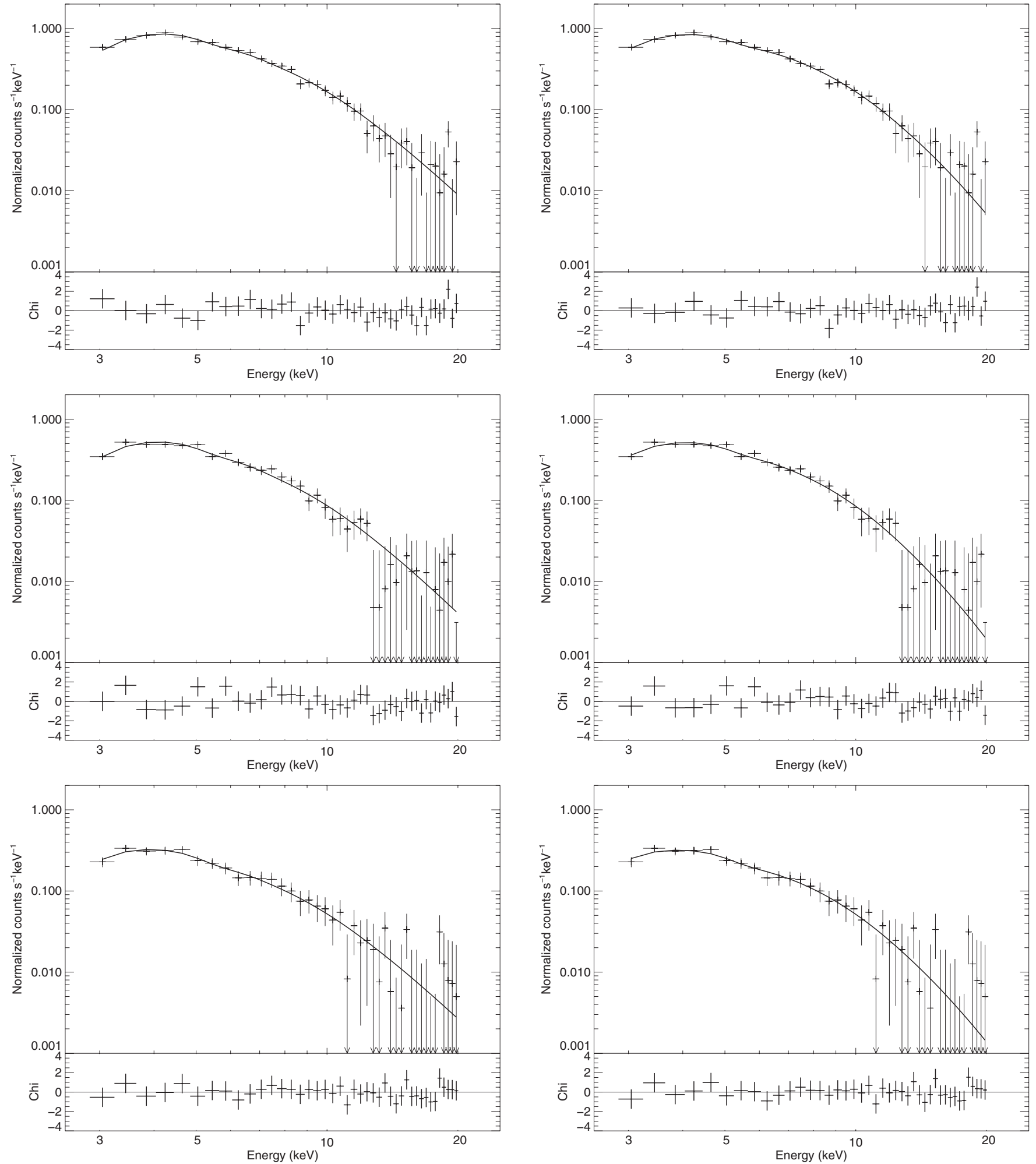

Figure 3. Power-law (left) and thermal bremsstrahlung (right) spectral fits to RXTE/PCA spectra for the three epochs of observing (2011 May 15, July 28, and August 22 , respectively). The lower panels in each show the number of standard deviations of the model from the data.

give the best-fit values from the reanalysis. While Motch et al. (1991) reported average EXOSAT values for the best-fit parameters for both power-law and thermal bremsstrahlung models, we report the individual observation values here (Tables 3 and 4). We found an average power-law index of 2.27 and an average electron temperature of $7.5 \mathrm{keV}$. No simultaneous fitting between different instruments was performed due to the variations in column density, flux, and power-law index. Consequently, the relative normalizations between the instruments are assumed to be unity.

\subsection{Correlations and Trends}

The present 2011 RXTE observations that occurred three, five, and six months after the last outburst (2011 February 23) show a clear steeply dropping flux (Figure 4). However, when the XMM-Newton value from 12 months after the outburst 

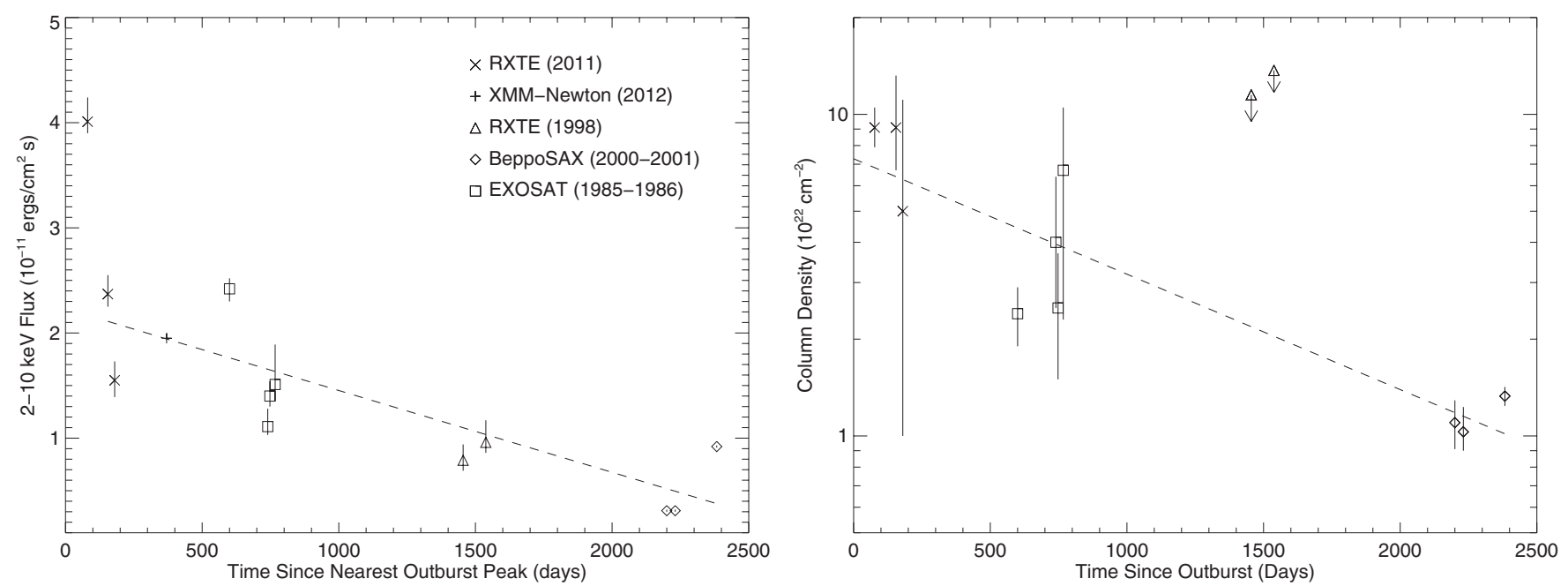

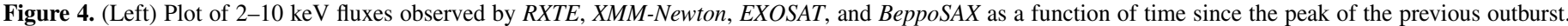

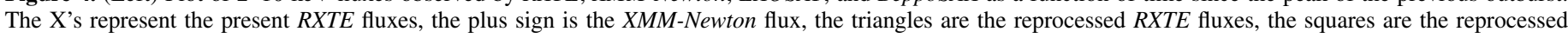

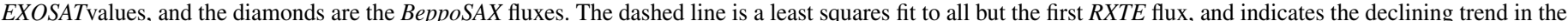

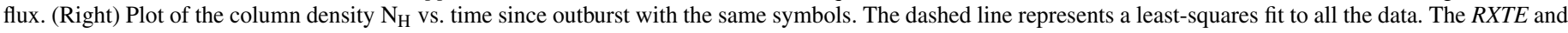
EXOSAT points are from the reanalysis reported here, and the BeppoSAX points are from Orlandini et al. (2004).

Table 3

Power-law Spectral Fit Results

\begin{tabular}{lcccc}
\hline \hline Date & $N_{\mathrm{H}}{ }^{\mathrm{a}}$ & Photon Index & Flux $^{\mathrm{b}}$ & $\chi_{v}^{2 \mathrm{c}}$ \\
\hline 2011 May 15 & $9.1_{-1.2}^{+1.4}$ & $2.40_{-0.07}^{+0.11}$ & $4.01_{-0.11}^{+0.23}$ & 0.67 \\
2011 Jul 28 & $9.1_{-2.4}^{+4.1}$ & $2.59_{-0.13}^{+0.24}$ & $2.37_{-0.12}^{+0.18}$ & 0.75 \\
2012 Aug 22 & $5.0_{-4.0}^{+6.1}$ & $2.44_{-0.28}^{+0.43}$ & $1.55_{-0.16}^{+0.18}$ & 0.42 \\
\hline 1998 Aug 21 $^{\mathrm{d}}$ & $<11.5$ & $2.30_{-0.60}^{+0.53}$ & $0.79_{-0.10}^{+0.15}$ & 0.50 \\
$1998 \mathrm{Nov} \mathrm{12}$ & $<13.7$ & $2.40_{-0.59}^{+0.63}$ & $0.96_{-0.10}^{+0.21}$ & 0.46 \\
\hline 1985 Sep 23 $^{\mathrm{e}}$ & $2.4_{-0.5}^{+0.5}$ & $1.83_{-0.07}^{+0.13}$ & $2.46_{-0.13}^{+0.09}$ & 1.33 \\
$1986 \mathrm{Feb} \mathrm{10}^{\mathrm{e}}$ & $4.0_{-1.5}^{+2.4}$ & $2.42_{-0.34}^{+0.50}$ & $1.18_{-0.11}^{+0.11}$ & 0.96 \\
$1986 \mathrm{Feb} \mathrm{18}$ & $2.5_{-1.0}^{+1.2}$ & $1.98_{-0.27}^{+0.27}$ & $1.44_{-0.11}^{+0.11}$ & 0.81 \\
$1986 \mathrm{Mar} 9 \mathrm{e}$ & $6.7_{-4.4}^{+3.8}$ & $2.85_{-1.00}^{+0.73}$ & $1.56_{-0.16}^{+0.34}$ & 0.89 \\
\hline
\end{tabular}

Notes.

a Column density in units of $10^{22} \mathrm{~cm}^{-2}$.

b Absorbed 2-10 keV power-law flux in units of $10^{-11} \mathrm{erg} \mathrm{cm}^{-2} \mathrm{~s}^{-1}$.

c $\chi^{2}$ per degree of freedom for 39 degrees of freedom for RXTE and 52 degrees of freedom for EXOSAT.

d Reanalysis of Negueruela et al. (2000) RXTE observations.

e Reanalysis of Motch et al. (1991) EXOSAT observations.

is added, the interpretation is less clear, and a flux level of $\sim 2 \times 10^{-11} \mathrm{erg} \mathrm{cm}^{-2} \mathrm{~s}^{-1} 6-12$ months after the outburst is indicated. The addition of the earlier RXTE, BeppoSAX, and EXOSAT observations performed 2-6 yr into quiescence sample the declining flux from 0.25 to $6 \mathrm{yr}$ after an outburst (Figure 4, left), albeit not the same outbursts and not all either normal or giant outbursts. The dashed line in Figure 4 (left) is a least squares fit to all but the first $R X T E$ point, and reflects a Pearson correlation coefficient of -0.785 (a null hypothesis probability of 0.012 ). If taken at face value, the quiescent flux values indicate a slowly declining quiescent level with a slope of $3.8 \times 10^{-12} \mathrm{erg} \mathrm{cm}^{-2} \mathrm{~s}^{-1}$ per day after these three outbursts. The 2011-2012 RXTE data show a slightly raised flux in the first observation, as do the EXOSAT data. This may be indicative of small levels of flaring during quiescence, which would be yet another indication of continuing, variable accretion. It may also be an effect of orbital phase in an isotropic stellar wind.
Table 4

Bremsstrahlung Spectral Fit Results

\begin{tabular}{lcccc}
\hline \hline Date & $N_{\mathrm{H}^{\mathrm{a}}}$ & $k T^{\mathrm{b}}$ & Flux $^{\mathrm{c}}$ & $\chi_{v}^{2 \mathrm{~d}}$ \\
\hline 2011 May 15 & $3.7_{-0.8}^{+1.8}$ & $8.22_{-0.95}^{+0.88}$ & $4.22_{-0.17}^{+0.19}$ & 0.60 \\
2011 Jul 28 & $3.7_{-1.6}^{+3.2}$ & $6.59_{-1.05}^{+0.97}$ & $2.48_{-0.14}^{+0.19}$ & 0.65 \\
2012 Aug 22 & $<5.4$ & $7.44_{-2.15}^{+2.30}$ & $1.62_{-0.17}^{+0.14}$ & 0.41 \\
\hline 1998 Aug 21 $^{\mathrm{e}}$ & $<7.0$ & $9.06_{-4.04}^{+8.13}$ & $0.80_{-0.09}^{+0.13}$ & 0.48 \\
1998 Nov 12 $^{\mathrm{e}}$ & $<8.1$ & $7.17_{-2.64}^{+6.62}$ & $1.00_{-0.11}^{+0.16}$ & 0.42 \\
\hline 1985 Sep 23 $^{\mathrm{f}}$ & $1.6_{-0.4}^{+0.8}$ & $11.14_{-2.19}^{+2.15}$ & $2.42_{-0.12}^{+0.10}$ & 1.25 \\
1986 Feb 10 $^{\mathrm{f}}$ & $2.4_{-1.1}^{+1.9}$ & $4.66_{-1.15}^{+3.33}$ & $1.11_{-0.08}^{+0.17}$ & 0.93 \\
1986 Feb 18 $^{\mathrm{f}}$ & $1.6_{-0.6}^{+1.1}$ & $8.42_{-2.53}^{+6.81}$ & $1.40_{-0.10}^{+0.14}$ & 0.81 \\
1986 Mar 9 $^{\mathrm{f}}$ & $4.1_{-2.8}^{+3.4}$ & $3.84_{-1.43}^{+8.26}$ & $1.51_{-0.16}^{+0.38}$ & 0.90 \\
\hline
\end{tabular}

Notes.

${ }^{a}$ Column density in units of $10^{22} \mathrm{~cm}^{-2}$.

${ }^{\mathrm{b}}$ Electron temperature in $\mathrm{keV}$.

${ }^{c}$ Absorbed 2-10 keV flux $\times 10^{-11} \mathrm{erg} \mathrm{cm}^{-2} \mathrm{~s}^{-1}$.

${ }^{\mathrm{d}} \chi^{2}$ per degree of freedom for 40 degrees of freedom for RXTE and 52 degrees of freedom for EXOSAT.

${ }^{\mathrm{e}}$ Reanalysis of Negueruela et al. (2000) observations.

${ }^{\mathrm{f}}$ Reanalysis of Motch et al. (1991) observations.

As the observed intensity decreased linearly as a function of time since outburst, the column density decreased exponentially (Figure 4, right). The Pearson correlation coefficient for the log of $N_{\mathrm{H}}$ versus time since outburst is -0.897 (a null hypothesis probability of 0.001 ). This naturally results in column density increasing with the $2-10 \mathrm{keV}$ power-law flux (Figure 5, right), and in this case the Pearson correlation coefficient is 0.772 (a null hypothesis probability of 0.015 ). Finally, the powerlaw index does not appear to correlate with the power-law flux (Figure 5, left).

\section{DISCUSSION AND CONCLUSIONS}

A $0535+26$ continues to show pulsations, as seen in most but not all of the observations. The fact that the first and brightest of the three new RXTE observations in quiescence did not exhibit detectable pulsations, while the second, $\sim 50 \%$ weaker 

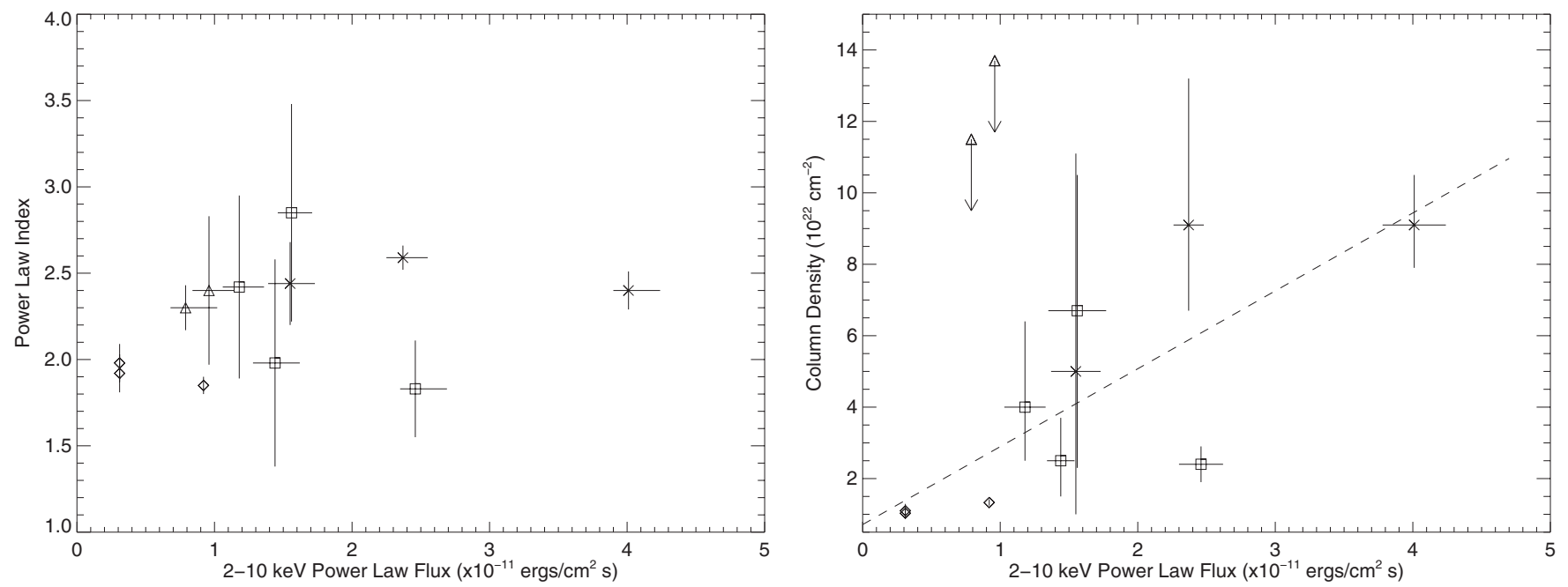

Figure 5. (Left) Plot of the power-law index vs. $2-10 \mathrm{keV}$ power-law flux. Note that no correlation is evident. $X$ 's represent the new $R X T E$ data, triangles the reanalyzed RXTE data, and squares the reanalyzed EXOSAT data. The diamonds represent the BeppoSAX data (Orlandini et al. 2004). (Right) Plot of the column density vs. $2-10 \mathrm{keV}$ power-law flux with the same symbols. The dashed line represents the least-squares fit to the data.

observation did with a similar live time, is strong evidence that the pulsations are not always present in quiescence. This would indicate in general that accretion onto the polar caps was still occurring, but that the accretion may not always be channeled to the magnetic poles. In quiescence the neutron star in A $0535+26$ is expected to be centrifugally inhibited (Campana et al. 2002), and plasma entry into the magnetosphere may be mediated by various instabilities affecting the extent of the emission region (Arons \& Lea 1976).

Okazaki \& Negueruela (2001) have modeled the outbursts from $\mathrm{Be} / \mathrm{X}$-ray binaries using a viscous decretion model, where the presence of outbursts depends upon the neutron star orbit with respect to various resonances of the gas in the system. If the neutron star's orbit is outside a given resonance, matter from that resonance will not flow onto the neutron star, whereas if it is within a given resonance, the matter will flow. Okazaki \& Negueruela (2001) state for A $0535+26$ that the disk is truncated at the 4:1 resonance when outbursts are detected, and that a slight decrease in viscosity or disk temperature would lower the disk radius to the 5:1 resonance and thus have the system go into dormancy. The observation that the quiescent luminosity is declining in the composite light curve (Figure 4) could imply that an accumulated mass at the corotation radius (Haigh et al. 2004) is being depleted over time. Then quiescence in A $0535+26$ would be characterized by reduced accretion from the magnetospheric boundary, while the bright outbursts would be characterized by high levels of accretion from the companion's circumstellar disk.

Another possibility is that the companion may have a stellar wind in addition to that from the circumstellar disk. This would mean that the system could transition from disk accretion to stellar wind accretion. If the disk were to recede, as the $\mathrm{H} \alpha$ observations imply in 1998 and 2011-2012 (Figure 1 of Camero-Arranz et al. 2012 and V. McBride 2012, private communication), one might expect the system to transition to spherical wind accretion dominating over that of the disk. If there is a sufficient stellar wind to provide wind accretion as in GX 301-2 (e.g., Suchy et al. 2012), then the generation of $\mathrm{X}$-rays and pulsing seen in quiescence might be explained. The very small number of quiescent data points after each outburst as a function of orbital phase precludes any test of variations with orbital phase, which might have been evidence of stellar wind accretion.

de Loore et al. (1984) adopt a wind mass loss rate for HDE 245770 of $10^{-8} M_{\odot} \mathrm{yr}^{-1}$ from measurements of UV resonance lines and infrared fluxes. Spherical Bondi accretion for a $1.4 M_{\odot}$ neutron star at a distance of 267 light-seconds ( $a \sin i$ for the A $0535+26$ system) and a $2000 \mathrm{~km} \mathrm{~s}^{-1}$ wind velocity at infinity (e.g., Table 1 in Kudritzki \& Puls 2000) would predict the mass accretion rate onto the neutron star to be $\sim 3 \times 10^{-5}$ of the wind mass loss rate. If the bolometric quiescent $\mathrm{X}$-ray luminosity is about twice the $2-10 \mathrm{keV}$ luminosity, or $2.5 \times 10^{34} \mathrm{erg} \mathrm{s}^{-1}$ for a distance of $2 \mathrm{kpc}$, then one would predict a wind mass loss rate of $10^{-9} M_{\odot} \mathrm{yr}^{-1}$, if the conversion efficiency of gravitational to $\mathrm{X}$-ray energy is 0.1 . This estimate is $10 \%$ of the de Loore et al. (1984) value, which may be reasonable if $90 \%$ of the wind mass loss is due to the circumstellar disk. Waters et al. (1988) predict a factor of $10^{4}$ for the difference in $\mathrm{X}$-ray luminosities between polar and equatorial mass flows for HDE $245770\left(10^{31} \mathrm{erg} \mathrm{s}^{-1}\right.$ versus $\left.10^{35} \mathrm{erg} \mathrm{s}^{-1}\right)$. If the orbit of the neutron star is inclined to the equator of the companion, and if the mass flow changes with angle from the pole, the neutron star may sample a mass flow that produces fluxes in the $10^{34} \mathrm{erg} \mathrm{s}^{-1}$ range.

Should we see orbital variations of the flux due to the eccentric orbit in the RXTE data that was taken within one orbital period? A $2000 \mathrm{~km} \mathrm{~s}^{-1}$ wind would predict at most a factor of $\sim 3$ variation in flux between the three RXTE phases. The RXTE fluxes are in agreement with this limited variation.

The spectra of A $0535+26$ (Tables 3 and 4 plus the results from BeppoSAX; Orlandini et al. 2004; Mukherjee \& Paul 2005) indicate a decreasing amount of absorption at the source as time since an outburst increases (Figure 4, right). When comparing the various values of $N_{\mathrm{H}}$ versus orbital phase (given in Table 2) it is clear that this material is present in the line of sight independent of orbital phase. Thus, it must be either all pervasive in the system (the wind?) or very close to the neutron star (a declining accumulation of matter at the corotation radius?). For the former, variations in $N_{\mathrm{H}}$ might imply a variable stellar wind density, which in turn would result in a correlated variable X-ray flux (Figure 5, right). For the latter, the disk might thin as the material is drained from it. This could be further evidence for 
a continual draining of the material trapped near the corotation radius (D'Angelo \& Spruit 2012).

The power-law index is independent of flux over the flux range of $1-4 \times 10^{-11} \mathrm{erg} \mathrm{cm}^{-2} \mathrm{~s}^{-1}$ (Figure 5). In addition, the powerlaw indices measured for all of the quiescent observations are significantly steeper $(\sim 2.5$ versus $<1)$ than those seen during outbursts (e.g., Caballero et al. 2007). A steeper power law would imply less Compton upscattering of lower energy photons due to either a thinning of the hot electron scattering plasma, or a cooling of the electron distribution. This may be further indication that the accretion mode is different between outburst and quiescence.

The pulse profile exhibited by the 2011 July $28 R X T E$ data is single peaked and somewhat sinusoidal, as has been seen in the past for observations made at quiescent luminosities, as noted above. Compton Gamma Ray Observatory/BATSE observations hint at a change in the pulse profile to the single peak variety that occurs below $10^{37}$ erg $\mathrm{cm}^{-2} \mathrm{~s}^{-1}$ (Bildsten et al. 1997). This change from the double peaked structure of the outburst pulse profiles possibly indicates a change in the accretion regime. For low luminosities the beam pattern is expected to be more pencil beam-like, and more like a fan beam at higher luminosities (Becker et al. 2012 and references therein).

\subsection{Conclusions}

RXTE observations of A $0535+26$ months after a giant outburst were combined with three other sets of multiple observations of A0535+26 from other X-ray observatories after other outbursts to reveal that they all had a similar (within a factor of two), or slowly decreasing flux level. At least half of the observations detected pulsations, indicating that accretion onto the magnetic poles was still occurring, but perhaps intermittently. Optical observations of $\mathrm{H} \alpha$ emission at the times of the measurements indicated that the 1998 and 2011 data were taken during times of a highly diminished Be star disk, allowing for the possibility of sources of accreted material other than that responsible for the outbursts. When combining all 13 observations as a function of time since the last outburst (albeit not the same outbursts), the quiescent flux appears to drop by a factor of 2 over $6.5 \mathrm{yr}$, or $\Delta F_{\mathrm{X}} \sim 10^{-11} \mathrm{erg} \mathrm{cm}^{-2} \mathrm{~s}^{-1}$ with small $(\times 2)$ flares or possible orbital variations present. Based upon very limited statistics, the line-of-sight column density decreases by a factor of a few over the combined $6.5 \mathrm{yr}$ of quiescence, while the power law remained relatively constant. The quiescent flux and subsequent, correlated line of sight column depth could be due to a draining of material builtup at the corotation radius or due to spherical accretion from an isotropic, but diminishing, component of the stellar wind. Further characterization of the quiescent mode of A $0535+26$ will require more observations over a range of times since the last outburst in order to see if the slow downward trend in the flux continues, and to see if one could detect the orbital variations in flux expected for wind accretion in the eccentric orbit.

We acknowledge the assistance and mentoring of Eric Michelsen concerning Lomb-Scargle analysis and its interpretation, along with use of his program. Thanks go out to Ron Remillard for supplying the weekly A $0535+26$ ASM data for the duration of the RXTE mission. These results were provided by the ASM/RXTE teams at MIT and at the RXTE SOF and GOF at NASA's GSFC. We thank Evan Smith for his excellent efforts in scheduling the RXTE observations. We thank ISSI for hosting and financing international group meetings on modeling and observations of cyclotron lines in high-mass X-ray binaries. We acknowledge the GSFC HEASARC for providing extracted spectra and response files for the EXOSAT observations that allowed for the reanalysis of the A0535+26 observations. This work was supported by the Bundesministerium für Wirtschaft und Technologie through DLR grant 50 OR 1113. We thank J. E. Davis for the slxfig module which was used to create some of the plots through out this paper. A.C.A. acknowledges the support from grants AYA2012-39303, SGR2009- 811, and iLINK2011-0303.

\section{REFERENCES}

Arnaud, K. A. 1996, in ASP Conf. Ser. 101, Astronomical Data Analysis Software and Systems, ed. G. Jacoby \& J. Barnes (San Francisco, CA: ASP), 17

Arons, J., \& Lea, S. M. 1976, ApJ, 210, 792

Becker, P. A., Klochkov, D., Schönherr, G., et al. 2012, A\&A, 544, 123 Bildsten, L., Chakrabarty, D., Chiu, J., et al. 1997, ApJS, 113, 367

Caballero, I. 2009, PhD thesis, Eberhard Karls Universität Tübingen

Caballero, I., Kretchmar, P., Santangelo, A., et al. 2007, A\&A, 465, 26

Camero-Arranz, A., Finger, M. H., Wilson-Hodge, C. A., et al. 2012, ApJ, 754,20

Campana, S., Stella, L., Israel, G. L., et al. 2002, ApJ, 580, 389

D'Angelo, C. R., \& Spruit, H. C. 2012, MNRAS, 420, 416

de Loore, C., Giovannelli, F., van Dessel, E. L., et al. 1984, A\&A, 141, 279

Finger, M. H., Wilson, R. B., \& Hagedon, K. S. 1994, IAU Circ., 5931

Giovannelli, F., \& Graziati, L. S. 1992, SSRv, 59, 1

Grundstrom, E. D., Boyajian, T. S., Finch, C., et al. 2007, ApJ, 660, 1398

Haigh, N. J., Coe, M. J., \& Fabregat, J. 2004, MNRAS, 350, 1457

Illarionov, A. F., \& Sunyaev, R. A. 1975, A\&A, 39, 185

Jahoda, K., Markwardt, C. B., Radeva, Y., et al. 2006, ApJS, 163, 401

Kalberla, P. M. W., Burton, W. B., Hartmann, D., et al. 2005, A\&A, 440, 775

Kudritzki, R.-P., \& Puls, J. 2000, ARA\&A, 38, 613

Larsson, S. 1996, A\&AS, 117, 197

Makino, F., Cook, W., Grunsfeld, J., et al. 1989, IAU Circ., 4769

Mihara, T., Nakajima, M., Yamamoto, T., et al. 2010, ATel, 2970

Motch, C., Stella, L., Janot-Pacheco, E., \& Mouchet, M. 1991, ApJ, 369, 490

Mukherjee, U., \& Paul, B. 2005, A\&A, 431, 667

Nagase, F., Hayakawa, S., Kunieda, H., et al. 1982, ApJ, 263, 814

Negueruela, I., Reig, P., Finger, M. H., \& Roche, P. 2000, A\&A, 356, 1003

Okazaki, A. T., \& Negueruela, I. 2001, A\&A, 377, 161

Orlandini, M., Bartolini, C., Campana, S., et al. 2004, NuPhS, 132, 476

Rosenberg, F. D., Eyles, C. J., Skinner, G. K., \& Willmore, A. P. 1975, Natur, 256,628

Sembay, S., Schwartz, R. A., Orwig, L. E., Dennis, B. R., \& Davies, S. R. 1990, ApJ, 351, 675

Steele, I. A., Negueruela, I., Coe, M. J., \& Roche, P. 1998, MNRAS, 297, L5

Suchy, S., Fürst, F., Pottschmidt, K., et al. 2012, ApJ, 745, 124

Tueller, J., Ajello, M., Barthelmy, S., et al. 2005, ATel, 504

Verner, E. M., Ferland, G. J., Korista, K. T., \& Yakovlev, D. G. 1996, ApJ, 465,487

Waters, L. B. F. M., Taylor, A. R., van den Heuvel, E. P. J., Habets, G. M. H. J., \& Persi, P. 1988, A\&A, 198, 200

Wilms, J., Allen, A., \& McCray, R. 2000, ApJ, 542, 914

Wilson-Hodge, C., Finger, M. H., Camero-Arranz, A., \& Connaughton, V. 2009, ATel, 2324 